\title{
Circadian effects of ionizing radiation on reproductive function and clock genes expression in male mouse
}

\author{
Fenju Qin ${ }^{1,2^{*}}$, Ningang Liu ${ }^{2 \dagger}$, Jing $\mathrm{Nie}^{2}$, Tao Shen ${ }^{1}$, Yingjie Xu' ${ }^{1}$, Shuxian $\mathrm{Pan}^{2}, \mathrm{Hailong} \mathrm{Pei}^{2}$ and \\ Guangming Zhou ${ }^{2 *}$
}

\begin{abstract}
Background: Exposure to the ionizing radiation (IR) encountered outside the magnetic field of the Earth poses a persistent threat to the reproductive functions of astronauts. The potential effects of space IR on the circadian rhythms of male reproductive functions have not been well characterized so far.

Methods: Here, we investigated the circadian effects of IR exposure (3 Gy X-rays) on reproductive functional markers in mouse testicular tissue and epididymis at regular intervals over a 24-h day. For each animal, epididymis was tested for sperm motility, and the testis tissue was used for daily sperm production (DSP), testosterone levels, and activities of testicular enzymes (glucose-6-phosphate dehydrogenase (G6PDH), sorbitol dehydrogenase (SDH), lactic dehydrogenase (LDH), and acid phosphatase (ACP)), and the clock genes mRNA expression such as Clock, Bmal1, Ror- $\alpha$, Ror- $\beta$, or Ror- $\gamma$.

Results: Mice exposed to IR exhibited a disruption in circadian rhythms of reproductive markers, as indicated by decreased sperm motility, increased daily sperm production (DSP), and reduced activities of testis enzymes such as G6PDH, SDH, LDH, and ACP. Moreover, IR exposure also decreased mRNA expression of five clock genes (Clock, Bmal1, Ror- $a$, Ror- $\beta$, or Ror- $\gamma$ ) in testis, with alteration in the rhythm parameters.
\end{abstract}

Conclusion: These findings suggested potential health effects of IR exposure on reproductive functions of male astronauts, in terms of both the daily overall level as well as the circadian rhythmicity.

Keywords: lonizing radiation, Circadian rhythm, Reproductive marker, Clock genes

\section{Background}

Outside the protection of the Earth's magnetosphere and atmosphere is a universe full of ionizing radiation (IR). Since solar particle events (SPEs) produce a transient elevation in the rate of IR exposure in space, these events are likely to pose the greatest biological dosimetry

\footnotetext{
*Correspondence: qinfenju@usts.edu.cn; gmzhou@suda.edu.cn ${ }^{\dagger}$ Fenju Qin and Ningang Liu contributed equally to this work. ${ }^{1}$ School of Chemistry and Life science, Suzhou University of Science and Technology, Suzhou 215009, China

${ }^{2}$ School of Radiation Medicine and Protection, Medical College of Soochow University, Suzhou 215123, China
}

contribution that threatens astronaut safety. Data from six SPEs (February 1956, November 1960, August 1972, August 1989, September 1989, and October 1989) have been used to model worst-case scenarios. From such models, it has been estimated that during a SPE, astronauts may be exposed to a skin dose of $14.5 \mathrm{~Gy}$, testicular dose of $3 \mathrm{~Gy}$, heart dose of $8 \mathrm{~Gy}$, brain dose of 1.6 Gy, and lens dose of 7.5 Gy $[1,2]$. These radiation doses could have clinically significant effects for the individual organs. Among various health risks induced by IR in space, fertility reduction or even permanent sterility is one of the most important barriers impeding plans for original author(s) and the source, provide a link to the Creative Commons licence, and indicate if changes were made. The images or other third party material in this article are included in the article's Creative Commons licence, unless indicated otherwise in a credit line to the material. If material is not included in the article's Creative Commons licence and your intended use is not permitted by statutory regulation or exceeds the permitted use, you will need to obtain permission directly from the copyright holder. To view a copy of this licence, visit http://creativecommons.org/licenses/by/4.0/. The Creative Commons Public Domain Dedication waiver (http://creativeco mmons.org/publicdomain/zero/1.0/) applies to the data made available in this article, unless otherwise stated in a credit line to the data. 
interplanetary travel [3]. As the key organ involved in maintaining male fertility, testis is a critical radiosensitive target that might be significantly impaired by IR exposure $[4,5]$. The toxic effects of IR interfere with normal spermatogenesis, leading to reduced sperm counts and defective sperm production [6]. In genitourinary cancer radiotherapy, exposure to IR may result in temporary destruction or complete cessation of spermatogenesis $[7,8]$. In the case of spaceflight, a recent On-orbit animal study has revealed that space radiation contributes to the degenerative changes in mouse seminiferous tubules, accompanied by a distinct downregulated sperm number and increased inflammatory level $[9,10]$. It is therefore suggested that space radiation may pose health risks to the reproductive system of male astronauts.

As a result of daily changes in the environment signals (cycles in light/darkness, feeding, rest/activity, and temperature fluctuations), most organisms have developed a biological circadian clock that can synchronize physiological and behavioral processes to the 24-h light-dark (LD) cycle. At the organ level, the circadian clock also functions in the regulation of the reproductive axis consisting of the hypothalamus, pituitary, and gonads [11]. Mutations of the clock gene may impair animal fertility, and electromagnetic or chemical exposure affects the secretion of the reproductive hormone testosterone by suppressing the expression of clock genes such as Ror- $\alpha$, Bmal1, and Clock [12, 13]. Additionally, a dose-dependent circadian phase-advancing effect has been observed in radiation-exposed organs and cells, indicating an effect of radiation on circadian rhythmic output [14-16]. However, whether IR exposure affects the diurnal rhythms of male reproductive function and its underline mechanism has not yet been investigated. In this study, we report the effects of IR on reproductive functions and their daily rhythms in terms of clock genes expressions in testis, to try to reveal chronotoxic characteristics of space IR on male astronauts.

\section{Materials and methods \\ Animals and irradiation procedure}

C57BL/ 6 wild-type male mice (6 weeks old) used in this study were provided by the laboratory animal center of Soochow University. The animals were maintained in strict accordance with the Institutional Animal Care and Use Committee guidelines of the University (the number approved by the committee, A201804134). They were housed in the specific pathogen-free barrier system facility maintained at $25 \pm 2{ }^{\circ} \mathrm{C}$ temperature, $50 \pm 5 \%$ relative humidity and $12 \mathrm{~h} \mathrm{L-D}$ cycles $(6: 00 \mathrm{a} . \mathrm{m}$. to 18:00 p.m.). Incandescent lamps (150-200 lux) were arranged at cage level to provide light during the light cycle. All animal handling operations during the dark cycle were carried out with the use of dim red light (0.1 lux). The animals were fed on commercial diet and water was provided ad libitum. Animals' circadian rhythmicity was screened by locomotor activity after adaptive feeding.

Male mice with circadian rhythmicity were randomly divided into 6 ionizing radiation (IR) groups (X-ray exposure, 36 animals) and 6 control groups (shamexposed mice, 36 animals). Each group of mice was treated at a specific time-point of a day, and the procedure of irradiation was carried out following Fig. 1 . The experimental groups were positioned in a chamber fixed to subjected to whole-body irradiation of 3 Gy X-rays at different Control Time (CT) hours: CT 3:00, CT 7:00, CT 11:00, CT 15:00, CT 19:00, and CT 23:00, respectively, by using a cabinet $\mathrm{X}$-ray generator (Faxitron, Wheeling, IL, USA) operated at $100 \mathrm{kVp}$
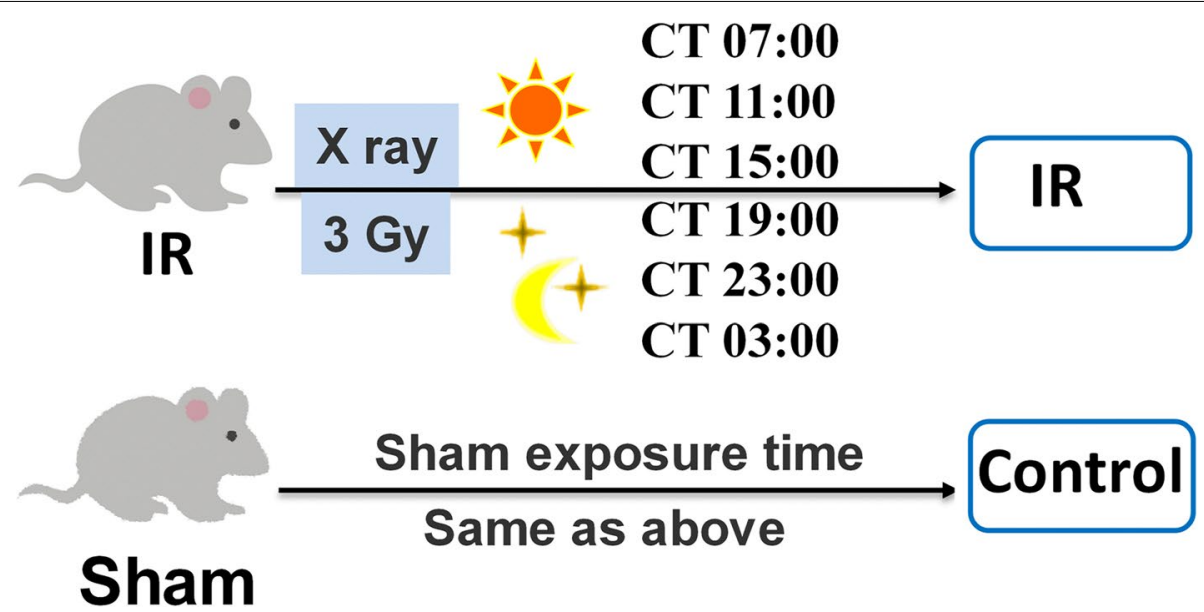

Fig. 1 Procedure of ionizing radiation for mice 
and $5 \mathrm{~mA}$ with a dose rate of $1.2 \mathrm{~Gy} / \mathrm{min}$. The thickness of the beryllium filter used in the X-ray generator was $0.25 \mathrm{~mm}$. Mice in the six control subgroups were handled in the same manner as those in radiation-exposed groups but without $\mathrm{X}$-ray. The mice were sacrificed under anesthesia by an intraperitoneal (i.p.) injection of chloral hydrate after the treatment [17]. Testes and epididymis were collected from each animal to record the weight. The right testis was examined for the testosterone levels and activity of testicular enzymes glucose-6-phosphate dehydrogenase (G6PDH), lactic dehydrogenase $(\mathrm{LDH})$, sorbitol dehydrogenase (SDH) and acid phosphatase (ACP), while the left testis was used for daily sperm production (DSP) and clock gene expression detection.

\section{Measurement of testosterone, G6PDH, SDH, LDH, and ACP}

From each mouse, the right testis was homogenized and centrifuged in saline $(0.9 \% \mathrm{NaCl}$ containing $0.05 \%$ Triton X-100) (Sigma-Aldrich, Shanghai, China), and the supernatant was used to determine the concentration of testosterone by a radioimmunoassay ELISA kit (Shanghai Yili Biotechnology Co. Ltd., Shanghai, China). A portion of the supernatant was used to measure the activities of G6PDH, SDH, LDH, and ACP by a commercially available kit (Nanjing Jiancheng Bioengineering Institute, Nanjing, China).

\section{Measurement of sperm production and motility}

The left epididymis of each mouse was excised and minced in a petri-dish in $2 \mathrm{ml}$ of warm Hank's solution (calcium and magnesium free) to obtain a free sperm suspension. The motility of sperm was recorded in forms of (a) rapid, progressive, and linear; (b) sluggish, progressive, and linear; (c) non-progressive; and (d) no mobility (WHO, 1992).

Daily sperm production (DSP) was determined as described by Joyce KL et al. (1993) [18]. Briefly, decapsulated testes were homogenized in ice-cold $0.9 \% \mathrm{NaCl}$ solution containing $0.01 \%$ Triton X-100. The homogenate was allowed to settle for $1 \mathrm{~min}$ and was gently mixed and stored on ice. The number of sperm heads was counted using a hemocytometer, and the number of spermatozoa produced per gram of testicular tissue per day was calculated using the following formula: Average count of sperm heads from four chambers $x \times$ square factor $x$ $\times$ hemocytometer factor $\times \times$ dilution factor divided by testis weight $(\mathrm{g})$ and the time (4.84 days) during spermatogenesis [19].

\section{mRNA expression of clock genes}

The other testis tissue was used to extract total RNA (high Pure RNA kit, Hoffmann-La Roche Ltd, Basel,
Switzerland) and the concentration was determined by the absorbance value at $260 \mathrm{~nm}$. The reverse transcription of $1 \mathrm{mg}$ RNA was carried out using the M-MLV reverse transcriptase Kit (Invitrogen Corporation, Carlsbad, CA). After incubation at $37{ }^{\circ} \mathrm{C}$ for $50 \mathrm{~min}$, M-MLV reverse transcriptase was inactivated by heating at $70{ }^{\circ} \mathrm{C}$ for $15 \mathrm{~min}$ and the genomic DNA were degraded by DNase I (Beyotime Inc., Nantong, China). Real-time PCR was performed utilizing the 7500 RealTime PCR System (Applied Biosystems Inc., Foster City, CA) and FastStart SYBR Green Master (Hoffmann-La Roche Ltd, Basel, Switzerland). In $10 \mathrm{ml} 2 \times \times$ Fast Start SYBR Green Mix we added 10pM of each primer set for Clock, Bmal1, Ror- $\alpha$, Ror- $\beta$, or Ror- $\gamma$ clock genes. The sequences of the primers used are shown in Table 1. The Real-time PCR was carried out in a AB step one plus $(\mathrm{AB})$ using the following thermal profile: initial PCR activation step at $95{ }^{\circ} \mathrm{C}$ for $2 \mathrm{~min}, 45$ cycles of denaturation at $95{ }^{\circ} \mathrm{C}$ for $20 \mathrm{~s}$, and annealing and extension at $60{ }^{\circ} \mathrm{C}$ for $40 \mathrm{~s}$. The fold-change for mRNA in mouse was calculated using the $2^{-\Delta \Delta \mathrm{Ct}}$ method. $\beta$-actin was used as an internal control. All samples were tested in triplicates.

\section{Statistical analysis}

Data presented in figures are mean \pm standard error. One-way analysis of variance (ANOVA) and the F-test were used to determine significant difference between groups. $p<0.05$ was considered statistically significant. Cosinor analysis was applied to fit data using the cosine software (Departments of Biology, School of Vhemistry and Life Science, Suzhou University of Science and Technology, Suzhou, Jiangsu, China). The $F$-test was used in the zero-amplitude test to analyze the significance of the circadian rhythm [20].

Table 1 The sequences of primers used in real-time PCR

\begin{tabular}{lll}
\hline Gene & & Primer sequence $\mathbf{5}^{\prime}$ to $\mathbf{3}^{\prime}$ \\
\hline Clock & Forward: & CGGCGAGAACTTGGCATT \\
& Reverse: & AGGAGTTGGGCTGTGATCA \\
Bmal1 & Forward: & GCAGTGCCACTGACTACCAAGA \\
& Reverse: & TCCTGGACATTGCATTGCAT \\
ROR- $a$ & Forward: & CCACCTACTCCTGTCCTCGTCAG \\
& Reverse: & CTTCTGCACCTCGGCGTACAAG \\
ROR- $\beta$ & Forward: & TGAGAATGTGTCGTGCCTTCAACC \\
& Reverse: & CAGAACAGCAGAGGAGAACAGAGC \\
ROR- $\boldsymbol{\gamma}$ & Forward: & GTCCAGACAGCCACTGCATTCC \\
& Reverse: & TGCCGTAGAAGGTCCTCCAGTC \\
$\beta-$ actin & Forward: & GCTCTTTTCCAGCCTTCCTTCTTG \\
& Reverse: & GTGTTGCATAGAGGTCTTTACGG \\
\hline
\end{tabular}


The data obtained were then fitted by the least squares method for cosinor analysis as expressed in the following Eq. (1).

$$
F(t)=M+A \cos (\omega t-\varphi)
$$

where $F$ stands for a fitted cosine function, $M$ is the median of the rhythm, $A$ represents the amplitude of the rhythm, $\omega$ is the radial frequency $(15 \% \mathrm{~h})$, and $\phi$ is the peak phase of the rhythm.

\section{Results}

Circadian rhythms of reproductive markers and clock gene expressions

The data of reproductive markers such as testicular enzymes activities and clock gene expressions were shown in Tables 2, 3, and 4, respectively. The parameters of cosinor analysis $(M, A, \omega$, and $\phi)$ were also shown in these tables.

Zero-amplitude test results of cosinor analysis of reproductive function data obtained from the control group were presented in Fig. 2a, b, and the straight lines and the cosinor fitting curves were presented in Figs. 3 and 4. By using zero-amplitude tests, the circles of confidence interval being not over-lapping with the pole (zero-point) suggested distinct circadian rhythms for the levels of three reproductive markers DSP, sperm motility, and testosterone (Fig. 2a), and the activities of four testicular marker enzymes ACP, G6PDH, SDH and LDH (Fig. 2b). From the fitting curves of cosinor analysis and the rhythm parameters in Figs. 3 and 4, the peak levels of DSP, sperm motility, testosterone, G6PGH, $\mathrm{SDH}, \mathrm{ACP}$, and LDH appeared at CT 5:27, CT 5:43, CT 6:15, CT 12:09, СТ 23:33, СТ 18:15, and CT 19:29, correspondingly.

The same analysis was used for clock gene temporal mRNA expression (Clock, Bmal1, Ror- $\alpha$, Ror- $\beta$ and Ror$\gamma$ ), the zero-amplitude test results revealed that the confidence interval circles of were not overlapped with the pole (Zero-point) to reject the zero-amplitude hypothesis, which indicated distinct rhythmic expression for these clock genes in mouse testis (Fig. 2c, d). Cosinor analysis showed the peak levels of Clock, Bmal1, Ror- $\alpha$, Ror- $\beta$, and Ror- $\gamma$ were at CT 5:38, CT 6:02, CT 18:55, CT 19:20, and CT 19:40, respectively (Fig. 5).

\section{Alteration in amplitude and peak time of reproductive markers and clock gene expressions induced by IR}

The straight lines and the cosinor fitting curves of reproductive markers and clock genes expression obtained from control and IR two groups were presented in Figs. 3, 4, and 5. Amplitude and peak time are the important parameters of daily rhythm oscillations period. As showed in Fig. 3a, the oscillation phase of DSP in IR treated mouse testes were antiphase to the control, the peak time of DSP was shifted from CT 5:27 to CT 17:31 after radiation exposure. Meanwhile, the amplitudes of DSP oscillations period became severe in IR exposed testes from 5.52 to $9.95(p<0.05)$. In contrast to the interconversion of DSP circadian phases in irradiated mice testis, the IR exposure does not affect significantly the phase distribution of other reproductive markers. Moreover, the amplitudes of testosterone levels and two testicular marker enzymes activities (SDH and LDH) were sharply shrunk after irradiation except ACP amplified $(p<0.05)$ (Figs. 3c and 4b-d).

For circadian rhythms of clock genes expression, the peak time shifted more than one hour only happened in Ror- $\alpha$, Ror- $\beta$ expression after IR exposure (Fig. $5 \mathrm{c}, \mathrm{d}$ ), the amplitudes shrunk or amplified significantly in all five clock genes expression $(p<0.05)$ (Fig. 5).

\section{Alteration in daily average level of reproductive markers and clock gene expressions induced by IR}

The variation in the daily average levels of reproduction markers and clock genes in IR exposed mice was calculated using the formula: $=\left(\left(\right.\right.$ Daily mean ${ }_{\mathrm{IR}}-$ Daily mean Control $/$ /Daily mean $\left.{ }_{\text {Control }}\right) \times 100 \%$. The results were presented in Table 5. IR exposure induced the changes in the daily mean values of the mice reproduction markers (including DSP, sperm motility, testosterone and G6PDH, SDH, ACP, and LDH) by $61.04 \%,-57.09 \%,-0.26 \%$, $5.22 \%,-29.82 \%,-11.42 \%$, and $-26.13 \%$, respectively. For these reproductive parameters after IR treatment, the most remarkable daily mean variation was found in DSP (from 11.19 to $18.0210^{6} / \mathrm{g} \times \mathrm{d}$ ) and sperm motility (from 64.41 to $27.64 \%$ ) (Fig. 3a, b). However, IR treatment made effective sperm number (DSP multiply by motility) reduced observably from 7.21 to $4.9810^{6} / \mathrm{g} \times \mathrm{d}$.

The alteration of daily average levels in gene expression of clock genes such as Clock, Bmal1, Ror- $\alpha$, Ror$\beta$, and Ror- $\gamma$ in exposed mice after IR is presented in Table 5 . The percentage changes of daily mean values of the clock genes expression happened in IR exposure mice by $-3.61 \%$ (Clock), $-63.49 \%$ (Bmal1), $-22.78 \%$ (Ror$\alpha$ ), 37.07 (Ror- $\beta$ ), and $-44.74 \%$ (Ror- $\gamma$ ), respectively. For the several clock genes, the most remarkable daily mean variation induced by IR treatment was found in Bmal1 expression from 0.63 to 0.23 (Fig. 5b).

\section{Circadian sensitivity of reproductive markers and clock gene expressions to IR}

As showed in Table 6, IR induced the different extent effects at six different circadian times: CT 3:00, CT 7:00, CT 11:00, CT 15:00, CT 19:00, or CT 23:00, respectively. Of the seven reproductive markers, the five parameters were increased or decreased at different timepoints, 


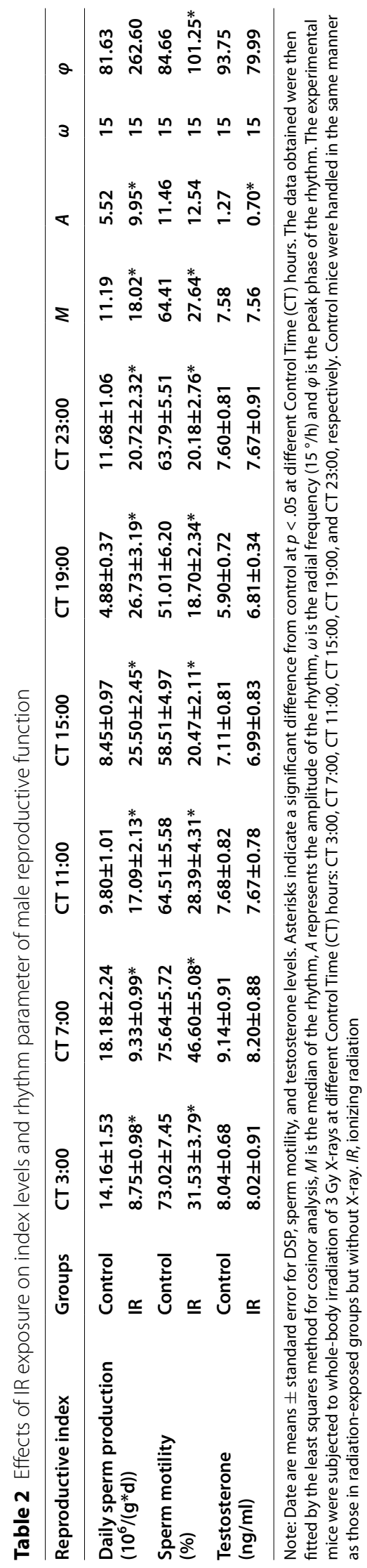




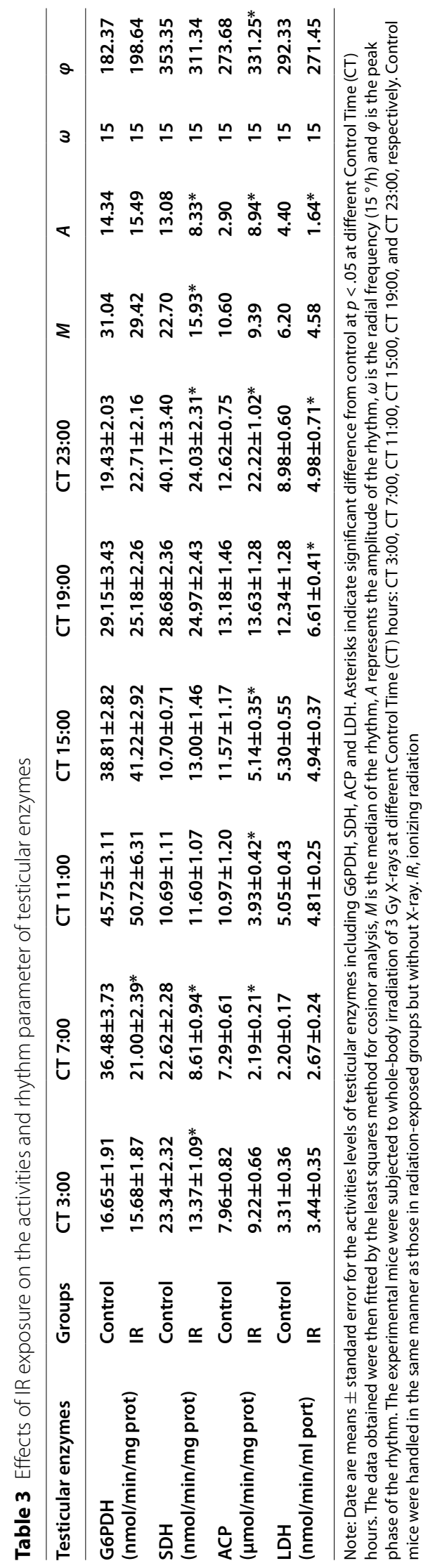




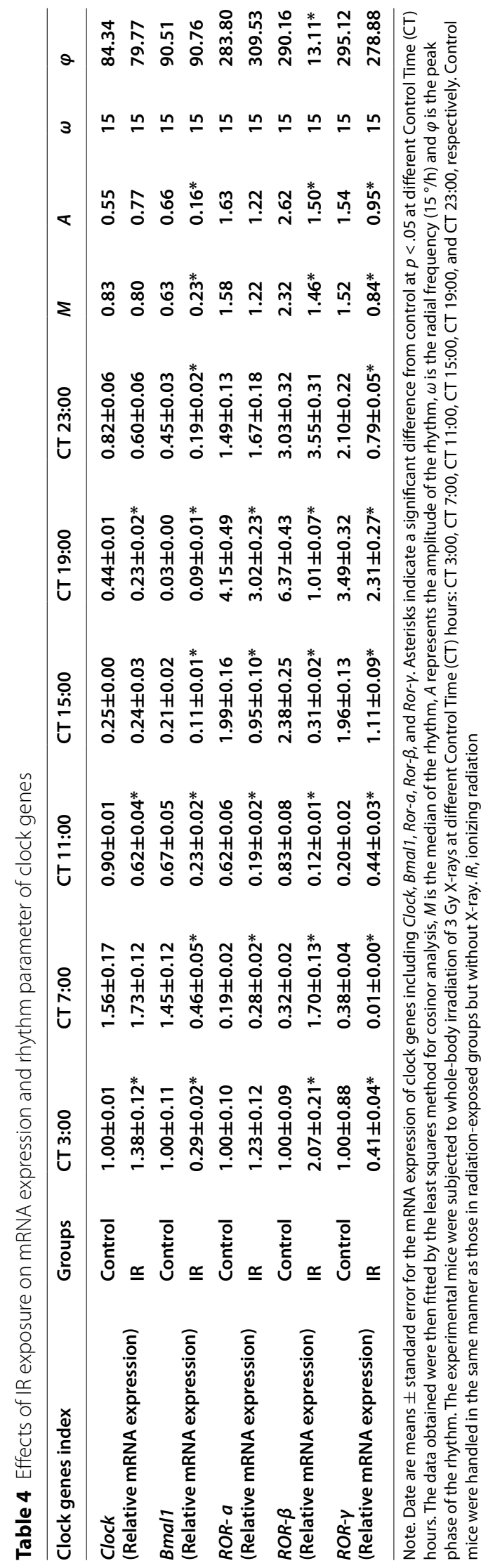



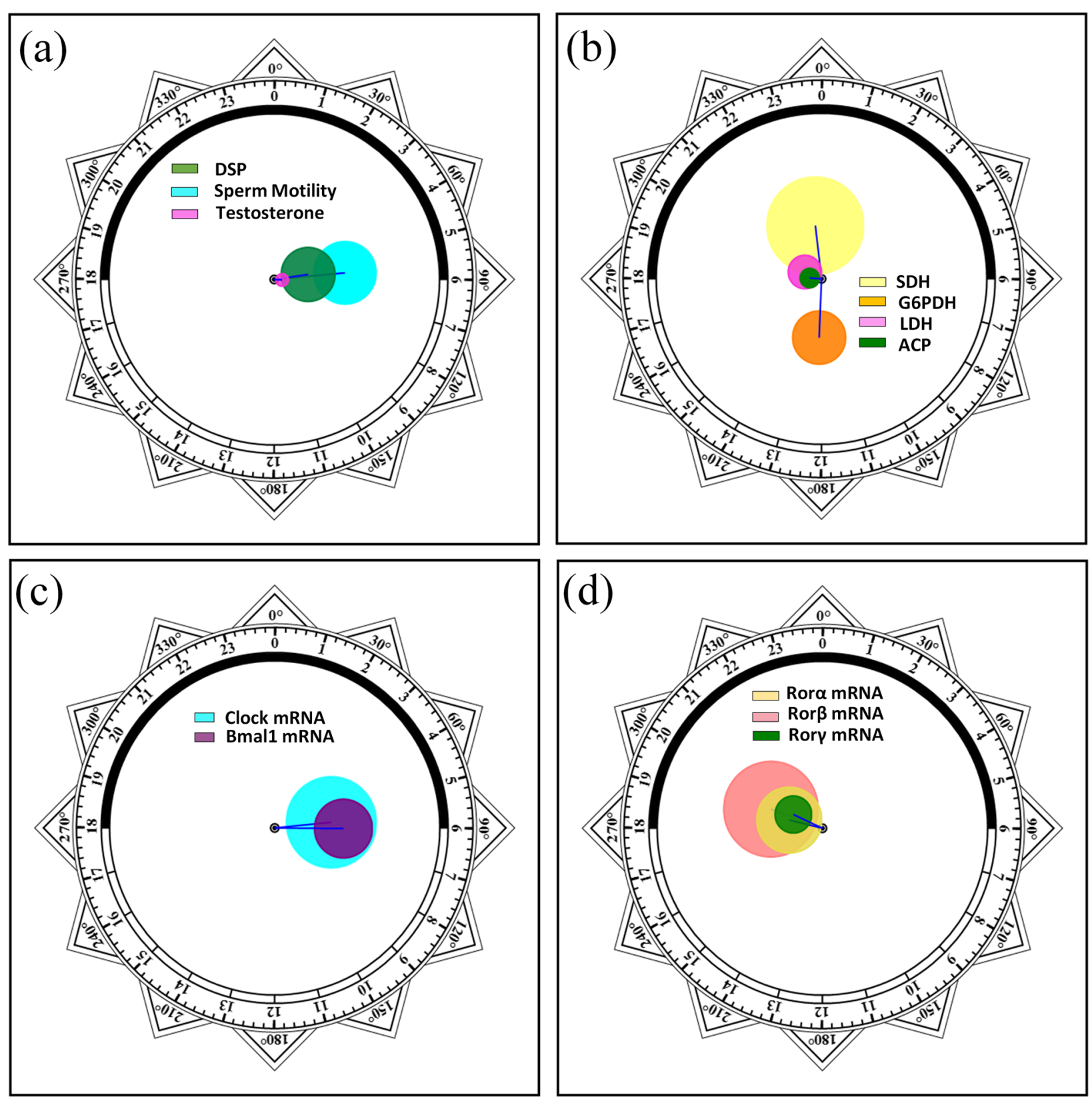

Fig. 2 Zero-amplitude test results for circadian rhythms in unirradiated control mice. a DSP, sperm motility, and testosterone; $\mathbf{b}$ G6PDH, SDH, ACP, and LDH; c Clock and Bmal1; and $\mathbf{d}$ Ror-a, Ror- $\beta$, and Ror- $\gamma$. The circles represent the 95\% joint confidence intervals of amplitude and acrophase. The pole (zero-point) of the polar coordinate was not overlapped by the circles, namely, the zero amplitude hypothesis was rejected because of $p<$ 0.05 , which indicates a distinct circadian rhythmicity of these reproductive markers and five clock genes expression

(See figure on next page.)

Fig. 3 Circadian rhythm of reproductive markers of Control and IR mice. The best-fitting curves (means \pm standard error) determined for DSP (a), sperm motility $(\mathbf{b})$, and testosterone $(\mathbf{c})$; $Y$-axis represents DSP, sperm motility and testosterone levels; $x$-axis represents the time during the $24-h$ light-dark cycle; $M$ and $A$ represents the median and the amplitude of the rhythm, respectively. Mice were exposed to X-ray (3 Gy) at CT 3:00, CT 7:00, CT 11:00, CT 15:00, CT 19:00, and CT 23:00 in a 24-h CT period. Control mice were in the same experimental circumstances without being exposed to $X$-ray. The white and dark boxes on the $x$-axis represent light and dark 

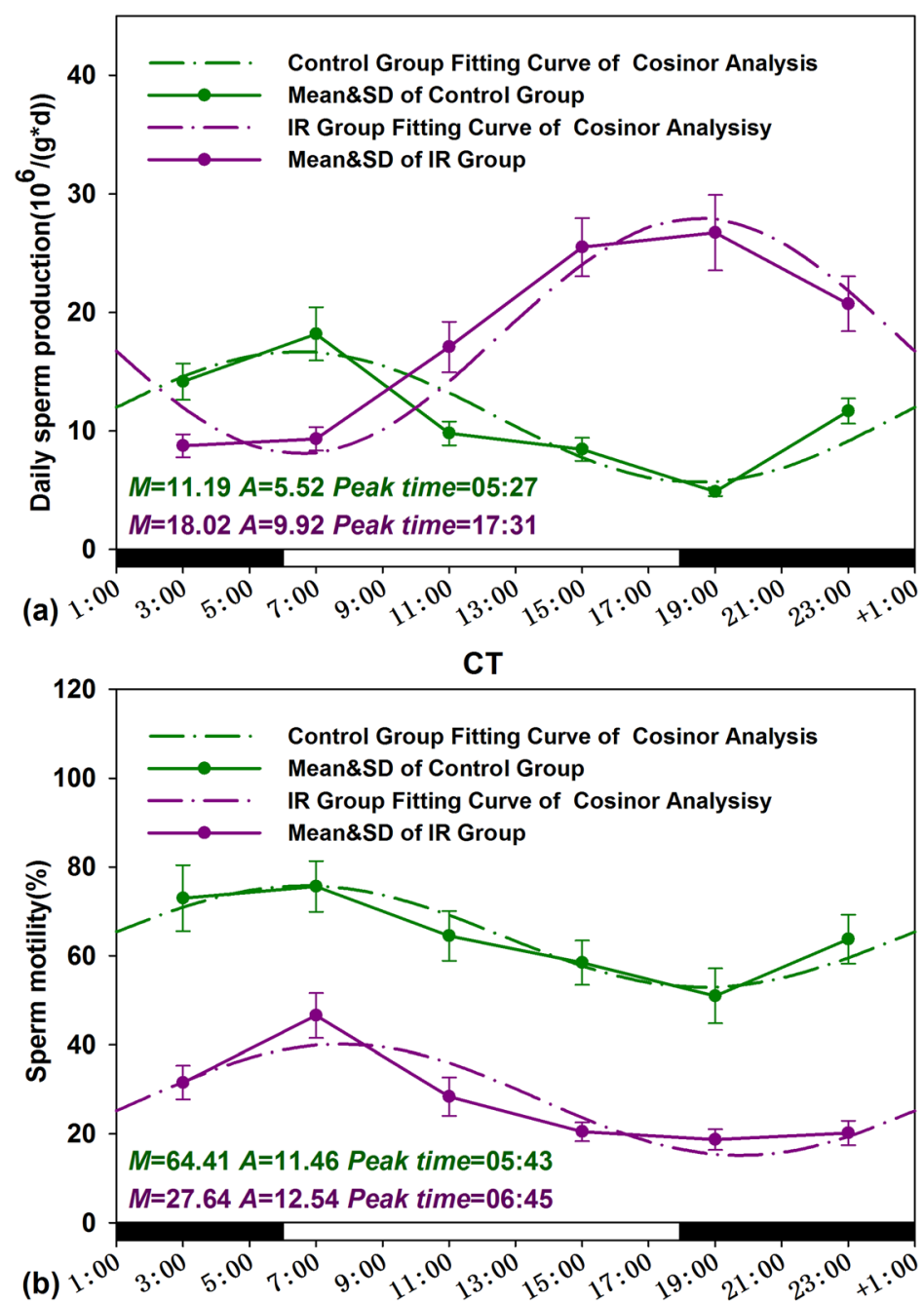

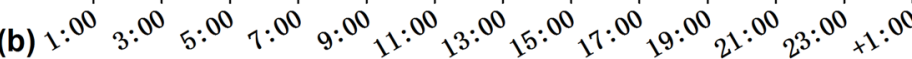

CT

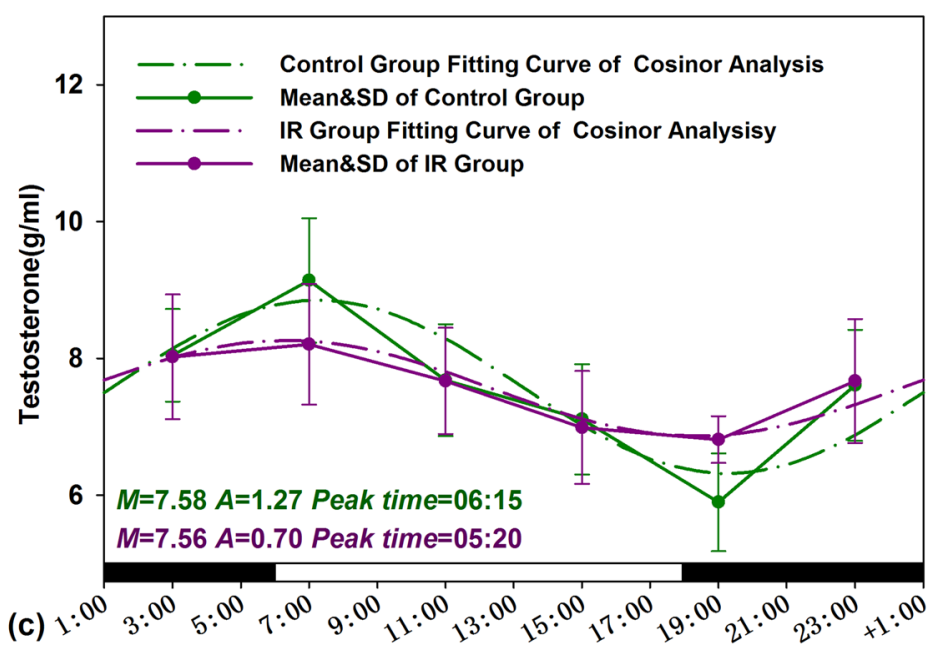

Fig. 3 (See legend on previous page.) 

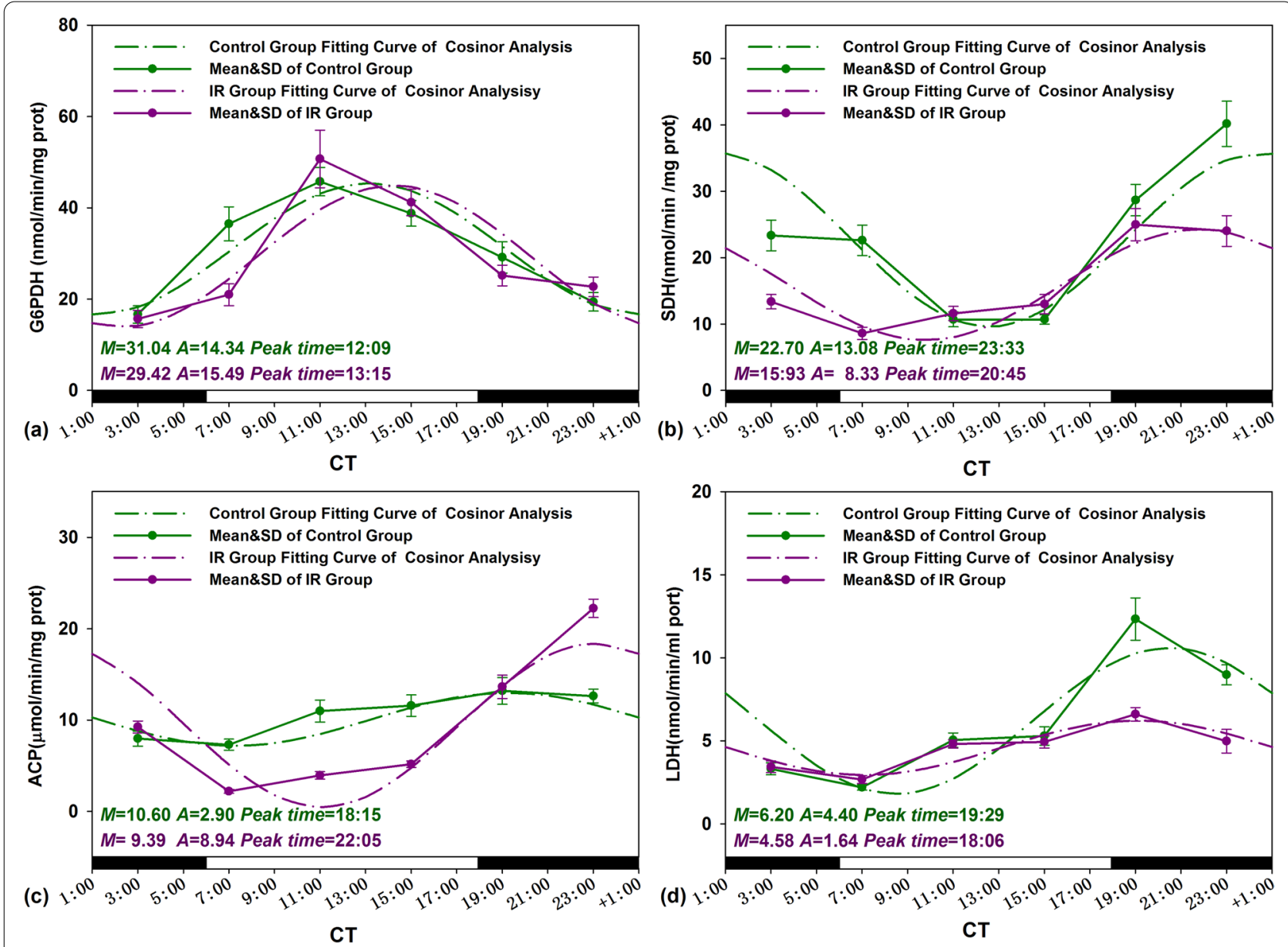

Fig. 4 Circadian rhythm of testis mark enzymes activities of Control and IR mice. The best-fitting curves (means \pm standard error) determined for $\mathrm{G} 6 \mathrm{PDH}(\mathbf{a}), \mathrm{SDH}(\mathbf{b}), \mathrm{ACP}(\mathbf{c})$, and LDH (d); $Y$-axis represents testis mark enzymes activities; $x$-axis represents the time during the 24-h light-dark cycle; $M$ and $A$ represents the median and the amplitude of the rhythm, respectively. Mice were exposed to X-ray (3 Gy) at CT 3:00, CT 7:00, CT 11:00, CT 15:00, CT 19:00, and CT 23:00 in a 24-h CT period. Control mice were in the same experimental circumstances without being exposed to X-ray. The white and dark boxes on the $x$-axis represent light and dark

except for sperm motility and LDH activities going down at all circadian timepoints. The most significant change of these reproductive markers happened by $-68.37 \%$ for sperm motility, $447.30 \%$ for DSP, $15.52 \%$ for testosterone, $-42.45 \%$ for G6PDH, $-61.94 \%$ for SDH, 76.05\% for ACP, and $-63.68 \%$ for LDH at CT 23:00, CT 19:00, CT 19:00, CT 7:00, CT 7:00, CT 23:00, and CT 19:00, respectively. For diurnal rhythm effect of clock genes, mRNA expression of Clock and Bmal1 most prominently changed by IR at CT $19: 00$ by $-46.33 \%$ and $206.90 \%$, respectively. The biggest variation of the other three clock gene expression induced by IR appeared at CT 11:00 for Ror-a, CT 7:00 for Ror- $\beta$, and CT 11:00 for Ror- $\gamma$ by $-69.09 \%$, 430.00\%, and $120.10 \%$, respectively (Table 6 ). The results showed that both male reproductive parameters and clock gene expression had their sensitive time points to IR.

\section{Discussion}

The mammalian circadian clock controls the timing of many biological processes. Reproductive physiology, particularly, is profoundly under the circadian regulation [21, 22]. Environmental cues such as light exposure can influence the timing of this internal clock system through the post translational modification of key components of the core molecular oscillator. During deep space exploration, radiation is one of the major challenges to astronauts. We have previously shown that radiofrequency radiation exposure can reset the circadian clock of the reproductive system in a time-of-day-dependent manner in male rats $[12,23]$. However, the potential effects of space IR on the circadian rhythms of male reproductive functions have not been well characterized so far. In the present study, we found a dramatic reproductive disruption concurrent with 


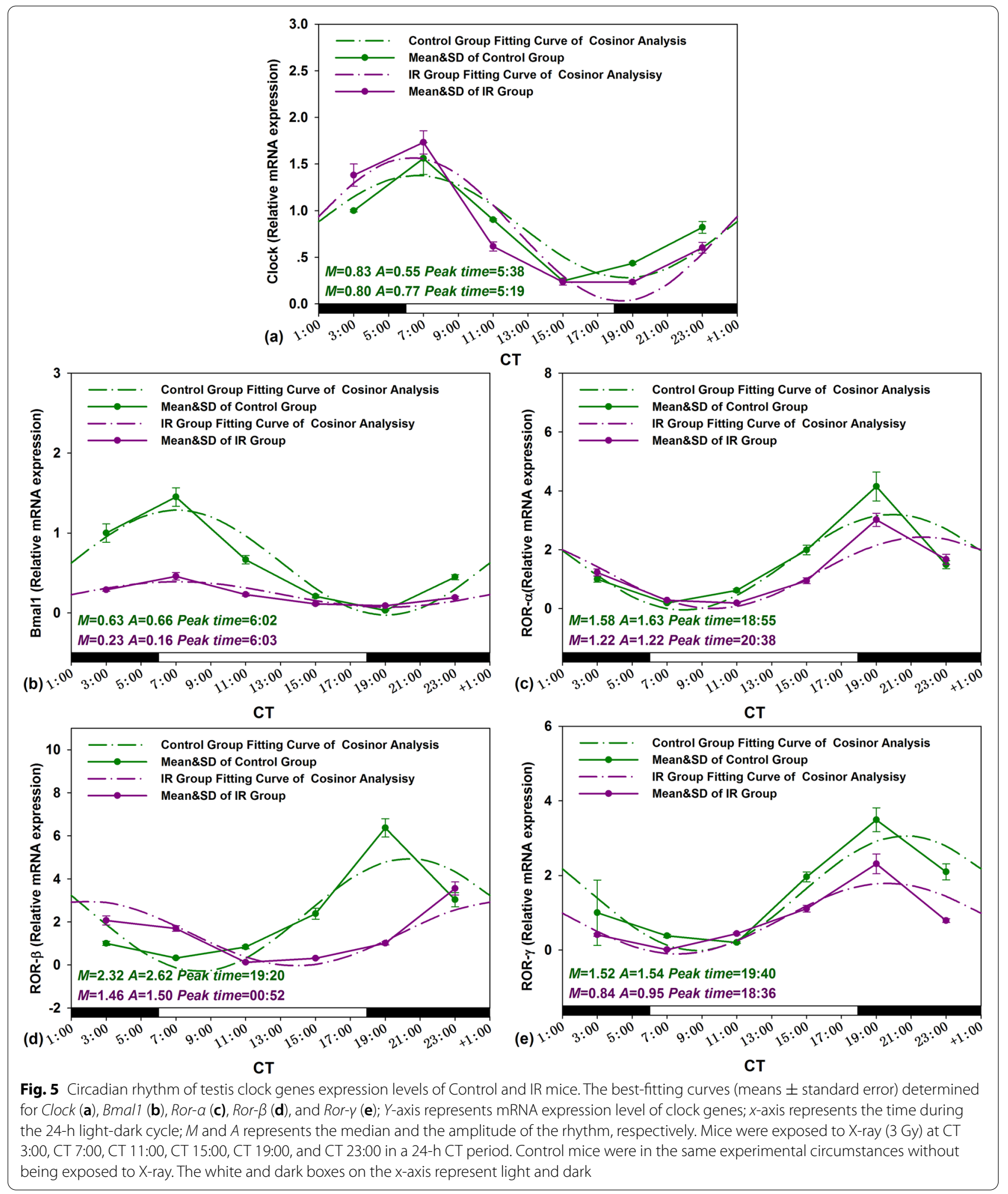

the decreased daily mean expression levels of five clock genes in the mice testes exposed to 3 Gy IR. The reproductive disruptions were induced by IR, in terms of reversed
DSP rhythm, and lower amplitudes of testosterone level, decreased SDH and LDH activities, and down-regulation of clock gene expressions. Furthermore, a significant 
Table 5 Percentage changes in the daily mean of male reproduction markers and clock genes expression between control and IR mice

\begin{tabular}{ll}
\hline Indicators & $\begin{array}{l}\text { Daily mean } \\
\text { changes } \\
(\%)\end{array}$ \\
\hline Daily sperm production & 61.04 \\
Motility & -57.09 \\
Testosterone & -0.26 \\
G6PDH & -5.22 \\
SDH & -29.82 \\
ACP & -11.42 \\
LDH & -26.13 \\
Clock & -3.61 \\
Bmal1 & -63.49 \\
ROR- $\alpha$ & -22.78 \\
ROR- $\beta$ & -37.07 \\
ROR- $\gamma$ & -44.74 \\
\hline
\end{tabular}

Note: $(\%)=\left(\left(\right.\right.$ Daily mean $_{\mathbb{R}}-$ Daily mean $\left.{ }_{\text {Control }}\right) /$ Daily mean $\left._{\text {Control }}\right) \times 100 \%$

timing effect of IR exposure on reproductive functional markers and clock gene expression was observed, when the changes between the IR exposed group and its sham control exposed group were compared at a specific corresponding time-point. These observations suggest that IR exposure can disrupt the reproductive function through resetting its circadian clock in mouse testis, indicating a significant role of the circadian clock in mediation of reproductive toxic effects of IR exposure.

Earlier studies have reported inconsistent results in the circadian expression of clock genes in normal mice testicles. While a nonrhythmic and developmental-stage specific expression of Cry1, Clock, and Ror- $\alpha$ in mice testicles was observed, more recent studies have revealed rhythmic expression of Per1, Per2, and Bmal1 genes in the testis of hamsters and mice [21, 24]. In agreement with these studies, our results showed the diurnal periodicity of productive functions in mice, with their level peak times at CT 5:27, CT 5:43, and CT 6:15 for three male reproductive markers (DSP, sperm motility, and testosterone, respectively), and the four enzymes
(G6PGH, SDH, ACP, and LDH) activities peak times at CT 12:09, CT 23:33, CT 18:15, and CT 19:29, respectively. To further understand whether the daily variation of male reproductive function is correlated with inherent rhythmic expression of clock genes in mouse testis, we examined the kinetics of clock gene expression in mRNA level and found that the expressions of all the five clock genes were oscillating throughout the day, with peaking times of Clock and Bmal1 gene at CT 5:38 and CT 6:02, and peaking times of Ror- $\alpha$, Ror- $\beta$, and Ror- $\gamma$ gene at CT 18:55, CT 19:20, and CT 19:40, respectively.

Ionizing radiation has been shown to induce tissue injury during space exposure to SPEs [25]. In this study, the simulated dose of 3 Gy IR obviously altered the daily mean level of sperm motility, a male reproductive marker that may be resulted from radiation-induced apoptosis and inflammation of the seminiferous tubules [7, 10, 2632]. Spermatogenesis is a high energy demanding process, and the activities of energy metabolism-related enzymes, including SDH and LDH, play key roles for the growth and development of testicular cells. For example, SDH, which is mainly found in chondriosome of Sertoli cells and spermatogenic cells, functions in pachytene spermatocyte maturation of germinal epithelium [33]. SDH is also responsible for providing energy to sperm cells by converting sorbitol to fructose. Similarly, LDH in Sertoli and spermatogenic cells also takes part in testis energy production and biotransformation. ACP, a lysosomal original enzyme in Sertoli cells of seminiferous tubules, has been reported to be involved in the development of spermatocytes and steroidogenesis, while G6PDH activities parallel the synthesis function of steroid hormone in Leydig cells $[19,34]$. In this study, the daily mean activities of G6PDH, SDH, LDH, and ACP were decreased after IR exposure, indicating the disruption in male reproductive functions induced by IR exposure.

There are many reports that the clock genes are involved in regulating male reproductive functions. Our previous work has shown that one of the clock genes, Ror- $\alpha$, plays a key role in testosterone biosynthesis through the StAR-P450 signal axis [12]. Epidemiological investigations have indicated associations of genetic

Table 6 Circadian effects of IR on reproductive markers and clock genes expression

\begin{tabular}{lllllllllllll}
\hline CT time & DSP & Sperm motility & Testosterone & G6PDH & SDH & ACP & LDH & Clock & Bmal & $R O R-a$ & $R O R-\beta$ & $R O R-\gamma$ \\
\hline $3: 00$ & -38.23 & -56.82 & -0.30 & -5.83 & -42.72 & 15.95 & -6.31 & 38.10 & -71.20 & 22.70 & 106.60 & -59.40 \\
$7: 00$ & -48.67 & -38.39 & -10.23 & -42.45 & -61.94 & -69.99 & -1.59 & 11.09 & -68.50 & 46.11 & 430.00 & -98.15 \\
$11: 00$ & 74.36 & -56.00 & -0.14 & 10.87 & 8.47 & -64.20 & -39.54 & -31.67 & -65.56 & -69.09 & -85.82 & 120.10 \\
$15: 00$ & 201.89 & -65.01 & -1.69 & 6.21 & 21.50 & -55.55 & -32.91 & -4.45 & -45.89 & -52.43 & -87.02 & -43.52 \\
$19: 00$ & 447.30 & -63.35 & 15.52 & -13.63 & -12.93 & 3.42 & -63.68 & -46.33 & 206.90 & -27.21 & -84.21 & -33.83 \\
$23: 00$ & 77.36 & -68.37 & 0.80 & 16.90 & -40.17 & 76.05 & -23.35 & -26.55 & -57.05 & 12.06 & 17.19 & -62.34
\end{tabular}


variability in the Clock gene with semen quality in idiopathic infertile men [35] and increased risk for male infertility [36, 37]. Mutations in the Clock gene can result in reduced male fertility in mouse [38, 39], and mutations in the Bmal1 gene altered the rate-limiting step of steroidogenesis that is a prerequisite for sperm production and maturation [40]. Thus, it is interesting to investigate whether IR-induced disruption of reproductive function is associated with altered clock gene expression. In this study, we demonstrated that the daily mean mRNA levels of all the five tested clock genes were significantly modified by IR exposure, which is consistent with the concurrent downregulation of reproductive functional marker enzymes in irradiated mice. These data raise the possibility that IR-induced suppression in daily mean expression of clock genes may be accounted for the subsequent reproductive function inhibition in mouse testes.

By using genetic circadian mutant mice models, recent studies have shown that the clock system is responsible to genotoxic stresses [41-43]. In support of these findings, IR exposure resulted in disrupted circadian oscillations in both DSP activity and clock gene expression level (Ror- $\alpha$ and Ror- $\beta$, indicated by peak time). These results reinforce the concept that for the clock to regulate reproductive functions, it must be responsive to environmental stress in time to optimally coordinate cellular metabolism with externally fluctuating metabolic demands [44, 45].

Our previous work has found that the circadian clock can significantly alter the DSP and sperm motility of rat, as well as the levels of antioxidants such as glutathione peroxidase (GSH-Px), superoxide dismutase (SOD), and melatonin, in response to radiofrequency radiation $[23,46]$. To follow up the work, this study is to make further efforts to explore the circadian effect of IR on reproductive functions, by treatment of mice to $\mathrm{X}$-ray irradiation at six different time points during the day (CT 3:00, CT 7:00, CT 11:00, CT 15:00, CT 19:00, and CT 23:00). As expected, our results did show some differential effects of IR at various circadian times. For example, changes in sperm motility and DSP were most significant in mice irradiated at CT 23:00 and CT 19:00, respectively, and the gene expressions of Ror- $\beta$ and Bmall were most upregulated in the same groups of mice irradiated at CT 7:00 and CT 19:00, respectively. These results indicated that not only IR itself, but the daily time at which radiation is exposed, may have a significant impact on the reproductive functions in a time-dependent manner.

\section{Conclusion}

Taken together, the present study provides with evidence of circadian adverse effects of IR on reproductive functions in male mouse. These effects are present in terms of both average daily levels of the reproductive markers, and circadian oscillation profiles of the clock genes expressions, which may be of value in the protection of astronauts in deep space exploration.

\section{Acknowledgements}

Thanks for the support from the NSFC, the Open Project Program of State Key Laboratory of Radiation Medicine and Protection.

\section{Authors' contributions}

FQ proposed study design, prepared manuscript, analyzed data, and do the whole experiments with JN, TS, YX, SP, and HP. NL analyzed data of molecular assays and prepared the manuscript, GZ modified manuscript, and proposed study design. All the authors read and approved the final manuscript.

\section{Funding}

The design, material, and analysis in this work were funded by the National Natural Science Foundation of China (81773463) and (82073480). The collection and detection were funded by the Open Project Program of State Key Laboratory of Radiation Medicine and Protection (GZK1201814).

\section{Availability of data and materials}

All data generated or analyzed during this study are included in this published article.

\section{Declarations}

Ethics approval and consent to participate

According to the guidelines and with ethical standards of the Animal Care \& Welfare Committee of Soochow University, this study was conducted, and all the procedures of animal experiments were reviewed and approved by the Committee.

\section{Consent for publication}

Not applicable.

\section{Competing interests}

The authors declare that they have no competing interests.

Received: 3 February 2021 Accepted: 24 September 2021

Published online: 11 October 2021

\section{References}

1. Squire T, Buchanan G, Elsaleh H. Mission to Mars: radiation safety or radiation disaster? Space transit and Mars radiation exposure risks-the shielding effect of a graphene space suit and a storm shelter during space travel. Int J Radiat Oncol. 2019;103:E44.

2. Pecaut MJ, Haerich P, Zuccarelli CN, Smith AL, Zendejas ED, Nelson GA. Behavioral consequences of radiation exposure to simulated space radiation in the C57BL/6 mouse: open field, rotorod, and acoustic startle. Cogn Affect Behav Neurosci. 2002;2:329-40.

3. Mishra B, Luderer U. Reproductive hazards of space travel in women and men. Nat Rev Endocrinol. 2019;15:713-30.

4. Silva AM, Correia S, Casalta-Lopes JE, Mamede AC, Cavaco JE, Botelho MF, et al. The protective effect of regucalcin against radiation-induced damage in testicular cells. Life Sci. 2016;164:31-41.

5. Khan S, Adhikari JS, Rizvi MA, Chaudhury NK. Radioprotective potential of melatonin against (6)(0)Co gamma-ray-induced testicular injury in male C57BL/6 mice. J Biomed Sci. 2015;22:61. 
6. Ding J, Wang H, Wu ZB, Zhao J, Zhang S, Li W. Protection of murine spermatogenesis against ionizing radiation-induced testicular injury by a green tea polyphenol. Biol Reprod. 2015;92:6.

7. Farhood B, Mortezaee K, Haghi-Aminjan H, Khanlarkhani N, Salehi E, Nashtaei MS, et al. A systematic review of radiation-induced testicular toxicities following radiotherapy for prostate cancer. J Cell Physiol. 2019;234:14828-37.

8. $\mathrm{Li} \mathrm{H}, \mathrm{HeY}$, Zhang H, Miao G. Differential proteome and gene expression reveal response to carbon ion irradiation in pubertal mice testes. Toxicol Lett. 2014;225:433-44.

9. Cancedda R, Liu Y, Ruggiu A, Tavella S, Biticchi R, Santucci D, et al. The Mice Drawer System (MDS) experiment and the space endurance recordbreaking mice. PLoS One. 2012;7:e32243.

10. Masini MA, Albi E, Barmo C, Bonfiglio T, Bruni L, Canesi L, et al. The impact of long-term exposure to space environment on adult mammalian organisms: a study on mouse thyroid and testis. PLoS One. 2012;7:e35418.

11. Sen A, Hoffmann HM. Role of core circadian clock genes in hormone release and target tissue sensitivity in the reproductive axis. Mol Cell Endocrinol. 2020;501:110655.

12. Qin F, Cao H, Yuan H, Guo W, Pei H, Cao Y, et al. 1800 MHz radiofrequency fields inhibits testosterone production via CaMKI /RORalpha pathway. Reprod Toxicol. 2018;81:229-36.

13. Qin F, Shen T, Cao H, Qian J, Zou D, Ye M, et al. CeO2NPs relieve radiofrequency radiation, improve testosterone synthesis, and clock gene expression in Leydig cells by enhancing antioxidation. Int J Nanomedicine. 2019;14:4601-11.

14. Muller MH, Rodel F, Rub U, Korf HW. Irradiation with X-rays phaseadvances the molecular clockwork in liver, adrenal gland and pancreas. Chronobiol Int. 2015;32:27-36.

15. Oklejewicz M, Destici E, Tamanini F, Hut RA, Janssens R, van der Horst GT. Phase resetting of the mammalian circadian clock by DNA damage. Curr Biol. 2008;18:286-91.

16. Forssell-Aronsson E, Quinlan RA. The Impact of circadian rhythms on medical imaging and radiotherapy regimes for the paediatric patient. Radiat Prot Dosimetry. 2017;173:16-20.

17. Nanwen Z, Wu W, Lin J. The comparative anesthesia study of different chloral hydrate concentrations in two kinds of male mice. J Clin Anesthesiol. 2014;30(2):171-3 In Chinese.

18. Joyce KL, Porcelli J, Cooke PS. Neonatal goitrogen treatment increases adult testis size and sperm production in the mouse. J Androl. 1993;14:448-55

19. Qin F, Shen T, Li J, Qian J, Zhang J, Zhou G, et al. SF-1 mediates reproductive toxicity induced by Cerium oxide nanoparticles in male mice. Journal of Nanobiotechnology. 2019;17:41.

20. Nelson W, Tong YL, Lee JK, Halberg F. Methods for cosinor-rhythmometry. Chronobiologia. 1979;6:305-23.

21. Sciarra F, Franceschini E, Campolo F, Gianfrilli D, Pallotti F, Paoli D, et al. Disruption of Circadian Rhythms: A crucial factor in the etiology of infertility. Int J Mol Sci. 2020;21:3943.

22. Yaw AM, Duong TV, Nguyen D, Hoffmann HM. Circadian rhythms in the mouse reproductive axis during the estrous cycle and pregnancy. J Neurosci Res. 2021;99:294-308.

23. Qin F, Zhang J, Cao H, Guo W, Chen L, Shen O, et al. Circadian alterations of reproductive functional markers in male rats exposed to $1800 \mathrm{MHz}$ radiofrequency field. Chronobiol Int. 2014;31:123-33.

24. Tong Y, Guo H, Brewer JM, Lee H, Lehman MN, Bittman EL. Expression of haPer1 and haBmal1 in Syrian hamsters: heterogeneity of transcripts and oscillations in the periphery. J Biol Rhythms. 2004;19:1131-25.

25. Cucinotta FA, Manuel FK, Jones J, Iszard G, Murrey J, Djojonegro $\mathrm{B}$, et al. Space radiation and cataracts in astronauts. Radiat Res. 2001;156:460-6.

26. Naeimi RA, Talebpour AF, Khalatbary AR, Ghasemi A, Zargari M, Ghesemi $M$, et al. Atorvastatin mitigates testicular injuries induced by ionizing radiation in mice. Reprod Toxicol. 2017;72:115-21.
27. Najafi M, Cheki M, Amini P, Javadi A, Shabeeb D, Eleojo MA. Evaluating the protective effect of resveratrol, Q10, and alpha-lipoic acid on radiationinduced mice spermatogenesis injury: A histopathological study. Int J Reprod Biomed. 2019;17:907-14.

28. Li HY, Zhang H, Miao GY, Xie Y, Sun C, Di CX, et al. Simulated microgravity conditions and carbon ion irradiation induce spermatogenic cell apoptosis and sperm DNA damage. Biomed Environ Sci. 2013;26:726-34.

29. Withers HR, Hunter N, Barkley HT Jr, Reid BO. Radiation survival and regeneration characteristics of spermatogenic stem cells of mouse testis. Radiat Res. 1974;57:88-103.

30. Rowley MJ, Leach DR, Warner GA, Heller CG. Effect of graded doses of ionizing radiation on the human testis. Radiat Res. 1974;59:665-78.

31. Zhao Q, Mao A, Yan J, Si J, Zhou R, Gan L, et al. Zhang H. (56)Fe ion irradiation induced apoptosis through Nrf2 pathway in mouse testis. Life Sci. 2016;157:32-7.

32. Jangiam $W$, Udomtanakunchai C, Reungpatthanaphong P, Tungjai M, Honikel L, Gordon CR, et al. Late effects of low-dose radiation on the bone marrow, lung, and testis collected from the same exposed BALB/CJ Mice. Dose Response. 2018;16:1559325818815031.

33. Mills NC, Means AR. Sorbitol dehydrogenase of rat testis: changes of activity during development, after hypophysectomy and following gonadotrophic hormone administration. Endocrinology. 1972;91:147-56.

34. Peruquetti RL, Taboga SR, Azeredo-Oliveira MT. Expression of acid phosphatase in the seminiferous epithelium of vertebrates. Genet Mol Res. 2010;9:620-8

35. Zhang J, Ding X, Li Y, Xia Y, Nie J, Yi C, et al. Association of CLOCK gene variants with semen quality in idiopathic infertile Han-Chinese males. Reprod Biomed Online. 2012;25:536-42.

36. Shen O, Ding X, Nie J, Xia Y, Wang X, Tong J, et al. Variants of the CLOCK gene affect the risk of idiopathic male infertility in the Han-Chinese population. Chronobiol Int. 2015;32:959-65.

37. Yang J, Zhang Z, Zhang Y, Zheng X, Lu Y, Tao D, et al. CLOCK interacts with RANBP9 and is involved in alternative splicing in spermatogenesis. Gene. 2018:642:199-204.

38. Liang $X$, Cheng $S$, Jiang $X$, He X, Wang Y, Jiang Z, et al. The noncircadian function of the circadian Clock gene in the regulation of male fertility. $J$ Biol Rhythms. 2013;28:208-17.

39. Dolatshad H, Campbell EA, O'Hara L, Maywood ES, Hastings MH, Johnson $\mathrm{MH}$. Developmental and reproductive performance in circadian mutant mice. Hum Reprod. 2006;21:68-79.

40. Alvarez JD, Hansen A, Ord T, Bebas P, Chappell PE, Giebultowicz JM, et al. The circadian clock protein BMAL1 is necessary for fertility and proper testosterone production in mice. J Biol Rhythms. 2008;23:26-36.

41. Koch CE, Leinweber B, Drengberg BC, Blaum C, Oster H. Interaction between circadian rhythms and stress. Neurobiol Stress. 2017;6:57-67.

42. Kuintzle RC, Chow ES, Westby TN, Gvakharia BO, Giebultowicz JM, Hendrix DA. Circadian deep sequencing reveals stress-response genes that adopt robust rhythmic expression during aging. Nat Commun. 2017;8:14529.

43. Oster $\mathrm{H}$. The interplay between stress, circadian clocks, and energy metabolism. J Endocrinol. 2020;247:R13-25.

44. Ramsey KM, Bass J. Circadian clocks in fuel harvesting and energy homeostasis. Cold Spring Harb Symp Quant Biol. 2011;76:63-72.

45. Jordan SD, Lamia KA. AMPK at the crossroads of circadian clocks and metabolism. Mol Cell Endocrinol. 2013;366:163-9.

46. Cao H, Qin F, Liu X, Wang J, Cao Y, Tong J, et al. Circadian rhythmicity of antioxidant markers in rats exposed to $1.8 \mathrm{GHz}$ radiofrequency fields. Int J Environ Res Public Health. 2015;12:2071-87.

\section{Publisher's Note}

Springer Nature remains neutral with regard to jurisdictional claims in published maps and institutional affiliations. 